\section{Pediatric glioma-associated KIAA1549:BRAF expression regulates neuroglial cell growth in a cell type-specific and mTOR-dependent manner}

\author{
Aparna Kaul, ${ }^{1,3}$ Yi-Hsien Chen, ${ }^{1,3}$ Ryan J. Emnett, ${ }^{1}$ \\ Sonika Dahiya, ${ }^{2}$ and David H. Gutmann ${ }^{1,4}$ \\ ${ }^{1}$ Department of Neurology, ${ }^{2}$ Department of Pathology \\ and Immunology, Washington University School of Medicine, \\ St. Louis, Missouri 63108, USA
}

Tandem duplications involving the BRAF kinase gene have recently been identified as the most frequent genetic alteration in sporadic pediatric glioma, creating a novel fusion protein (f-BRAF) with increased BRAF activity. To define the role of $\mathrm{f}-\mathrm{BRAF}$ in gliomagenesis, we demonstrate that f-BRAF regulates neural stem cell (NSC), but not astrocyte, proliferation and is sufficient to induce glioma-like lesions in mice. Moreover, f-BRAF-driven NSC proliferation results from tuberin/Rheb-mediated mammalian target of rapamycin (mTOR) hyperactivation, leading to S6-kinase-dependent degradation of p27. Collectively, these results establish mTOR pathway activation as a key growth regulatory mechanism common to both sporadic and familial low-grade gliomas in children.

Supplemental material is available for this article.

Received July 10, 2012; revised version accepted October 10, 2012.

In the pediatric population, the most common solid tumors originate in the brain, where they can be associated with significant morbidity. Among the histological subtypes, low-grade pilocytic astrocytomas (PAs) are the most frequently encountered brain tumors (Central Brain Tumor Registry of the United States 2012). PAs arise either sporadically or in the context of the Neurofibromatosis type 1 (NF1) inherited cancer disposition syndrome. Tumors arising in children with NF1 typically develop along the optic pathway, following biallelic inactivation of the NF1 tumor suppressor gene in neuroglial cells. The NF1 protein (neurofibromin) is a negative regulator of the RAS proto-oncogene such that neurofibromin loss in neuroglial cells results in increased RAS-dependent mammalian target of rapamycin (mTOR) signaling (Lee et al. 2010).

In contrast, sporadic PAs do not harbor mutations in the NF1 gene (Kluwe et al. 2001) but rather are associated with alterations in the $B R A F$ gene locus (Pfister et al. 2008). High-resolution genomic arrays and fluorescent in situ

[Keywords: pilocytic astrocytoma; astrocytes; neural stem cells]

${ }^{3}$ These authors contributed equally to this work.

${ }^{4}$ Corresponding author

E-mail gutmannd@neuro.wustl.edu

Article published online ahead of print. Article and publication date are online at http://www.genesdev.org/cgi/doi/10.1101/gad.200907.112. hybridization (FISH) analysis have identified tandem duplications on chromosome $7 \mathrm{q} 34$ that create novel genes containing the kinase domain of $B R A F$ fused with a fragment of the KIAA1549 gene. Subsequent studies have confirmed that the KIAA1549:BRAF fusion $(f-B R A F)$ is found in $65 \%-$ $75 \%$ of sporadic PAs (Jones et al. 2008), leading to elevated mitogen-activated protein kinase (MAPK) activation.

While it has been established that the presence of f-BRAF is a hallmark of sporadic PAs with diagnostic potential, its precise role in neuroglial cell growth and gliomagenesis is unresolved. Although f-BRAF can transform NIH3T3 cells (Jones et al. 2008), previous studies have demonstrated that BRAF does not increase astrocyte proliferation (Jacob et al. 2009), induces senescence of astrocytes (Jacob et al. 2011) and neural stem cells (NSCs) in vitro (Raabe et al. 2011), and does not result in gliomagenesis when ectopically expressed in mouse brains in vivo (Robinson et al. 2010).

In this study, we sought to define the role of this signature genetic alteration in neuroglial cell function and glioma formation. We demonstrate that f-BRAF regulates neuroglial cell growth in a cell type-specific manner via tuberous sclerosis complex (TSC)/Rheb activation of mTOR and is sufficient to induce glioma-like lesions in vivo.

\section{Results and Discussion}

The discovery of the signature KIAA1549:BRAF genetic alteration represents a major advance in our understanding of the molecular pathogenesis of sporadic PA. However, subsequent studies have raised important questions regarding the role of this potential driver mutation in neuroglial cell proliferation and PA tumorigenesis. Moreover, prior studies have examined the impact of wild-type or oncogenic BRAF (BRAF ${ }^{\mathrm{V} 600 \mathrm{E}}$ ) expression on astrocyte or astroglial progenitor function, rather than the signature PAassociated BRAF fusion protein. To determine whether f-BRAF expression is sufficient to confer a growth advantage to neuroglial progenitor cells, we expressed f-BRAF in cerebellar NSCs, since the majority of sporadic PAs arise in the cerebellum (Ohgaki and Kleihues 2005). Primary NSC cultures generated from postnatal day 1 (P1) mouse cerebella expressed the Sox2 stem cell and Olig2 glial progenitor markers, but not glial fibrillary acidic protein (GFAP; astrocyte) (Supplemental Fig. S1A), and were capable of multilineage differentiation (Supplemental Fig. S1B). Cerebellar NSCs were transduced with either empty $p B A B E$-puro vector $(p B A B E)$ or $f-B R A F$. Since BRAF activates MAPK in a MEK-dependent manner, a constitutively active MEK mutant $\left(M E K^{\mathrm{Q} 56 P}\right.$; caMEK) was included. Following caMEK or f-BRAF expression in cerebellar NSCs, there was increased MAPK activation (1.5-fold and twofold increased Thr202/Tyr204 phosphorylation, respectively) (Fig. 1A) and increased NSC proliferation, as assessed by secondary neurosphere diameters $(28 \%$ and $46 \%$ increases, respectively), direct cell counting (1.5-fold increase) (Fig. 1B) and limiting dilution analysis $(37 \pm 7$ pBABE-transduced vs. $2 \pm 1$ f-BRAF-transduced cells) (Supplemental Fig. S1C).

Oncogenic BRAF has been reported to induce activation of the mTOR pathway in melanoma, thyroid, and breast carcinomas (Babchia et al. 2010; Duong et al. 2012; Faustino et al. 2012). In addition, previous studies from our laboratory have demonstrated that the NF1 gene product neurofibromin regulates murine astrocyte, NSC, and glioma growth in an mTOR-dependent manner 
A

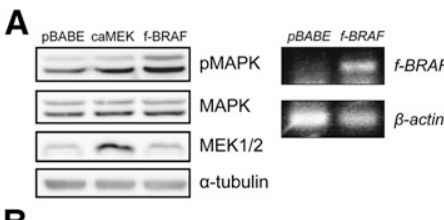

B

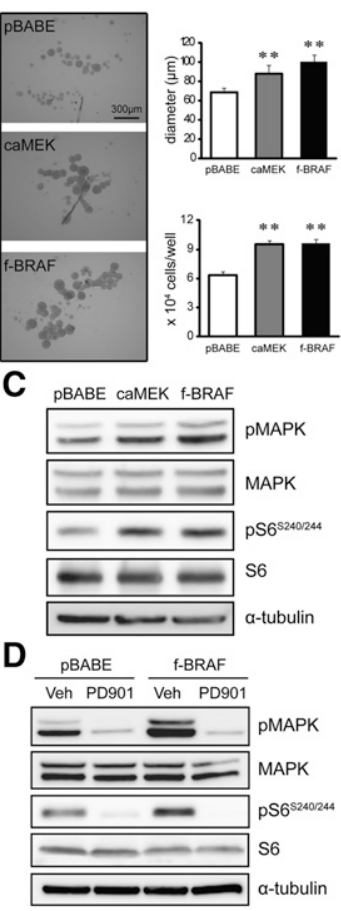

E

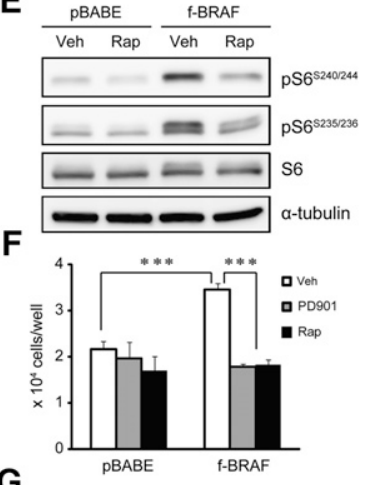

G

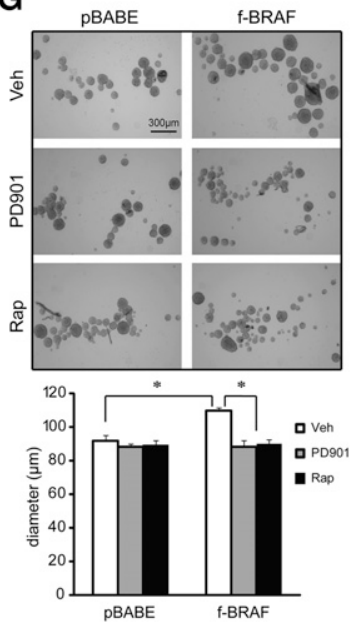

Figure 1. f-BRAF expression induced NSC proliferation and $m$ TOR pathway activation. (A) Increased MAPK phosphorylation was observed following caMEK and f-BRAF expression in cerebellar NSCs. $f-B R A F$ transgene expression was confirmed by RT-PCR. Increased NSC proliferation $(B)$ and S6 phosphorylation $(C)$ were observed in caMEK- and f-BRAF-expressing NSCs. S6 phosphorylation was attenuated following treatment with $2 \mathrm{nM}$ PD0325901 (PD901) (D) and $0.2 \mathrm{nM}$ rapamycin $(\operatorname{Rap})(E) .(F, G)$ PD901 and Rap restore f-BRAFexpressing NSC proliferation to control (pBABE) levels. $\alpha$-Tubulin served as an internal loading control. (Veh) Vehicle. Error bars denote mean \pm SD. Bars, $\left.300 \mu \mathrm{m} .\left(^{\star}\right) P<0.05 ;\left.\right|^{* \star}\right) P<0.01 ;\left(^{\star \star \star}\right) P<0.001$.

(Dasgupta et al. 2005; Lee et al. 2010), raising the intriguing possibility that both NF1-associated and sporadic $\mathrm{PA}$ share $\mathrm{mTOR}$ pathway activation as a major mitogenic driver. Consistent with this prediction, f-BRAF expression in cerebellar NSCs resulted in increased phosphorylation (activation) of ribosomal S6 protein (5.8-fold increase in Ser240/244, shown in Fig. 1C; 6.2-fold increase in Ser235/236, shown in Supplemental Fig. S1D) and S6kinase (S6K) (Thr389 shown in Supplemental Fig. S1E). Increased MAPK (1.6-fold) and S6 (1.7-fold) activation was also observed following BRAF ${ }^{\mathrm{V} 600}$ expression in cerebellar NSCs, with an accompanying increase in NSC proliferation $(23 \%$ increase in neurosphere diameter and 1.5-fold increase by direct cell counting) (Supplemental Fig. S1F).

We next sought to define how f-BRAF activates mTOR signaling. First, similar to f-BRAF, caMEK expression in NSCs resulted in S6 activation (Fig. 1C). Second, MEK inhibition with PD0325901 (PD901) reduced MAPK and S6 activation in both wild-type and f-BRAF-expressing NSCs (Fig. 1D) but only inhibited the proliferation of f-BRAFexpressing NSCs (Fig. 1 F,G). Third, rapamycin-mediated

mTOR inhibition attenuated the f-BRAF-induced increases in S6 activation (Fig. 1E) and proliferation (Fig. $1 F, G)$, similar to that observed following PD901 treatment. Finally, whereas MEK inhibition blocked f-BRAF-induced mTOR activation, rapamycin had no effect on f-BRAFinduced MAPK activation (Supplemental Fig. S1G). Collectively, these results demonstrate that f-BRAF regulates NSC proliferation by activating the mTOR pathway in a MEK-dependent manner.

Several studies have shown that ectopic expression of either RAF or BRAF alone is not sufficient to induce gliomas in mice (Lyustikman et al. 2008; Robinson et al. 2010; Gronych et al. 2011), suggesting that BRAF may not be a driver mutation. However, BRAF expression was targeted to progenitor cells in the cerebral hemispheres rather than the more typical locations (cerebellum, optic pathway, and brainstem) where $f-B R A F$-containing PAs arise in children. To establish a causative role for the signature $B R A F$ alteration in gliomagenesis, we show that $\mathrm{f}$-BRAF expression resulted in a fivefold increase in cerebellar NSC soft agar colony formation (Supplemental Fig. S2A). Next, mCherry-expressing cerebellar NSCs infected with control (pBABE) or f-BRAF were injected into the cerebella of 3-wk-old wild-type mice and analyzed at 2.5 and $6 \mathrm{mo}$ post-transplantation. At $2.5 \mathrm{mo}$, we observed threefold increases in the numbers of $\mathrm{Ki}-67^{+}$and $\mathrm{Iba} 1^{+}$cells at the injection sites in mice transplanted with f-BRAFexpressing NSCs $(n=3)$ relative to control virus-infected NSCs $(n=3)$. In addition, there was only a modest increase in cellularity and GFAP immunoreactivity and no nuclear atypia or cells with piloid morphologies (Supplemental Fig. S2B,C). However, at 6 mo post-injection, mice transplanted with f-BRAF-expressing NSCs $(n=5)$ harbored clear areas of increased cellularity near the injection sites, consistent with glioma-like lesions, including abnormal cell clusters with pale nuclei (Fig. 2A, H\&E, inset), increased $\mathrm{GFAP}^{+}$cells with piloid morphologies and elongated hair-like cytoplasmic processes (Fig. 2A, inset), increased numbers of proliferating $\left(\mathrm{Ki} 67^{+}\right)$cells (Fig. 2A,B), increased microglial (Iba1 ${ }^{+}$cells) infiltration (Fig. 2A,B), and increased numbers of endoglin ${ }^{+}$endothelial cells (data not shown) relative to pBABE-containing NSC-injected control mice $(n=4)$. Whereas mCherry ${ }^{+}$cells were identified at the injection sites from both pBABE- and f-BRAFtransduced NSC-transplanted mice (Fig. 2A), both NSCderived astrocytes (GFAP and mCherry double-positive cells) (Supplemental Fig. S2E) and host-derived astrocytes $\left(\mathrm{GFAP}^{+}\right.$only) (Supplemental Fig. S2E) were detected in the f-BRAF-expressing lesions. Consistent with the in vitro results, there was also increased pMAPK and pS6 immunostaining in f-BRAF-expressing NSC-engrafted mice at both 2.5 and 6 mo of age (Fig. 2C; Supplemental Fig. S2D). While no gross neurological deficits or changes in survival were observed, body weight was reduced by $28 \%$ in mice injected with f-BRAF-expressing NSCs.

The finding that f-BRAF expression in cerebellar NSCs generated glioma-like lesions in the cerebellum following engraftment likely reflects differences in the innate capabilities of these brain region-specific NSCs. In this regard, previous studies from our laboratory have revealed NSC regional specificity in response to $f-B R A F$ expression and $N f 1$ gene loss. Whereas lateral ventricle NSCs exhibit marginal or no increase in NSC proliferation following Nf1 inactivation or f-BRAF expression, respectively, third ventricle and brainstem NSCs demonstrate increased proliferation and 


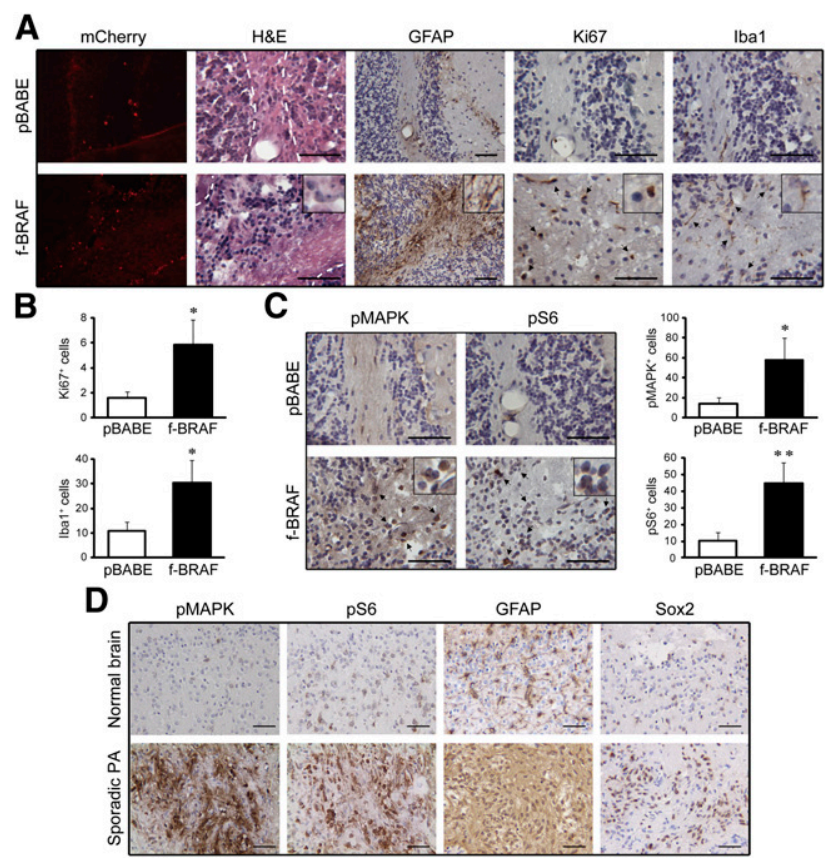

Figure 2. f-BRAF induced glioma-like lesions in mice, and mTOR activation was observed in human PAs. (A) Injection sites (dotted lines) contain mCherry ${ }^{+}$pBABE- or f-BRAF-expressing cells. Abnormal cell clusters with pale nuclei (insets, $\mathrm{H} \& \mathrm{E})$, increased number of GFAP-immunoreactive cells with piloid morphologies (inset), increased $\mathrm{Ki} 67^{+}$cells (arrows and inset; see also B, top), and increased $\mathrm{Iba}^{+}$cells (arrows and inset; see also $B$, bottom) were observed in f-BRAF-induced lesions. $(C)$ Increased numbers of $\mathrm{pMAPK}^{+}$and $\mathrm{pS}^{+}$ cells (arrows) were found in f-BRAF-induced lesions. $(D)$ Representative images show increased pMAPK and pS6 staining, along with increased GFAP and Sox2 immunoreactivity, in f-BRAF-containing human PAs. A normal human brain was used as a reference control. Error bars denote mean $\pm \mathrm{SD}$. $\left(^{\star}\right) P<0.05 ;\left(^{\star \star}\right) P<0.01$. Bars, $50 \mu \mathrm{m}$.

gliogenesis (Lee et al. 2010, 2012). Consistent with prior BRAF results (Raabe et al. 2011) and our recent findings regarding NSC brain region heterogeneity (Lee et al. 2012), no change in neocortex NSC proliferation was seen following f-BRAF expression (Supplemental Fig. S2F).

To determine whether sporadic human PAs likewise exhibit increased mTOR activation, we used a tissue microarray (TMA) containing 54 sporadic PAs. Consistent with the in vitro findings linking MAPK and mTOR activation in murine NSCs, a strong correlation was observed between mTOR activation (pS6 immunostaining) and MAPK activation (pMAPK immunostaining; $\chi^{2}$ test) (Supplemental Fig. S2G). Since the BRAF genotyping was not available for the PAs contained in the TMA, we analyzed 14 sporadic PA cases in which the precise $f-B R A F$ mutation was known and found that all f-BRAF-containing PA tumors examined had increased pMAPK and pS6 immunoreactivity relative to normal brains (Fig. 2D; Supplemental Fig. S2H). In addition, these tumors exhibited diffuse GFAP expression and contained a smaller population of cells with Sox2 expression (Fig. 2D; Supplemental Fig. S2H).

To define the mechanism by which f-BRAF activates mTOR in cerebellar NSCs, we performed several experiments based on prior studies in other cell types, demonstrating that MAPK can activate mTOR by several mechanisms, including $90-\mathrm{kDa}$ ribosomal protein S6 kinase (RSK) activation and inactivation of TSC protein function (Ma et al. 2005; Carriere et al. 2008). First, we showed that
f-BRAF expression does not increase RSK phosphorylation (Thr573), excluding RSK-mediated TORC1 activation as the mechanism underlying f-BRAF growth regulation (Fig. 3A). Second, we observed a 2.6-fold increase in tuberin phosphorylation (Ser939) following f-BRAF expression in NSCs (Fig. 3A). Similarly, there was a 2.9-fold and 1.6-fold increase in tuberin phosphorylation in NSCs after caMEK and $\mathrm{BRAF}^{\mathrm{V} 600}$ expression, respectively (Supplemental Fig. S3A,B). Moreover, treatment of f-BRAF-expressing NSCs with PD901 reduced the observed increases in tuberin and S6 phosphorylation (Fig. 3B), demonstrating that f-BRAFinduced tuberin phosphorylation was MEK-dependent.

Since prior reports on BRAF growth regulation in astrocytes have yielded conflicting results, we transduced primary GFAP ${ }^{+}$but Sox2/Olig2-negative astrocytes from P1-P2 mouse cerebella (Supplemental Fig. S3C) with $p B A B E, B R A F^{V 600}$ or $f-B R A F$. Consistent with the known effects of BRAF on MAPK activation, both BRAF ${ }^{\mathrm{V} 600}$ and f-BRAF expression increased MAPK phosphorylation (6.5fold and 4.4-fold, respectively), but neither BRAF molecule increased astrocyte proliferation (Fig. 3C). The inability of BRAF to increase astrocyte proliferation was not the result of induced cellular senescence or programmed cell death (TUNEL labeling) (Supplemental Fig. S3D). Similarly, this failure to increase cell proliferation was also observed in another astrocyte population (forebrain) despite increased MAPK activation (Supplemental Fig. S3E). Moreover, the inability of BRAF to increase astrocyte proliferation was not dependent on mitogen (EGF, PDGF, or PACAP) availability (Supplemental Fig. S3F). Consistent with the finding that BRAF-induced MAPK activation is dependent on MEK activity (Supplemental Fig. S3G), caMEK expression also did not increase primary astrocyte proliferation (Supplemental Fig. S3H). Together, these results demonstrate that f-BRAF increases neuroglial cell proliferation in a cell typespecific manner unrelated to its ability to activate MEK/ MAPK signaling. This observed cell type specificity underscores the critical cellular context in which cancerassociated driver mutations must occur in order to facilitate proliferation, transformation, and tumorigenesis. The differential response of NSCs and astrocytes to f-BRAF expression is consistent with previously reported roles for neuroglial progenitors in gliomagenesis (Taylor et al. 2005; Alcantara Llaguno et al. 2009; Liu et al. 2011).

To elucidate the mechanism underlying this cell typespecific effect, we compared the status of mTOR pathway activation following f-BRAF expression in astrocytes and NSCs. While f-BRAF expression in NSCs resulted in an 8.6fold increase in S6 phosphorylation, there was no change in S6 phosphorylation following f-BRAF expression in astrocytes (Fig. 3D). Furthermore, there was no change in tuberin phosphorylation following f-BRAF expression in astrocytes (Fig. 3E), indicating that MAPK, although activated by f-BRAF, was unable to phosphorylate and inactivate tuberin and thus could not activate mTOR in astrocytes.

We next sought to define the potential mechanism underlying differential tuberin phosphorylation in NSCs relative to astrocytes. Following hypotonic lysis fractionation, there was $\sim 13$-fold more tuberin in the membrane fraction of astrocytes compared with NSCs following normalization to total tuberin in each fraction (Fig. 3F). These results support a model in which cell type-specific f-BRAF-mediated mTOR activation reflects the subcellular localization of tuberin. Polycystin-1, an integral membrane protein, suppresses mTOR activation by directly interacting with 
A

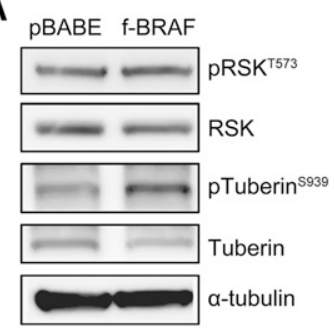

B

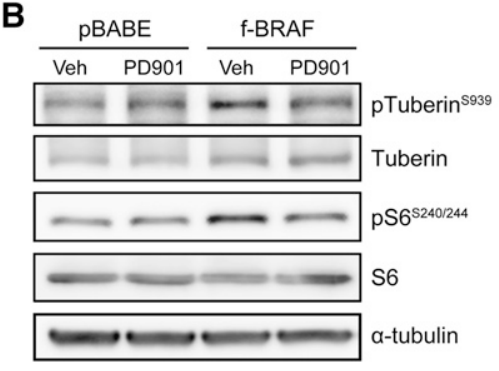

C

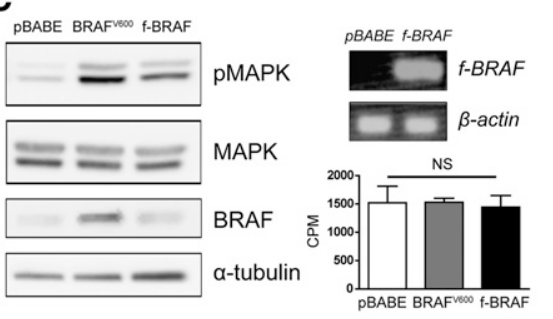

Figure 3. Cell type-specific effects of f-BRAF expression on NSC proliferation and tuberin phosphorylation. (A) Increased tuberin phosphorylation was observed in f-BRAFexpressing NSCs, with no change in RSK phosphorylation. (B) f-BRAF-induced tuberin and S6 phosphorylation was inhibited by PD901 treatment. $(C)$ Increased MAPK phosphorylation was observed in $\mathrm{BRAF}^{\mathrm{V} 600}$ - and $\mathrm{f}$-BRAF-expressing astrocytes. $f-B R A F$ transgene expression was verified by RT-PCR. Compared with the vector control, BRAF expression did not increase astrocyte proliferation. $(D)$ Increased S6 phosphorylation was observed in f-BRAF-expressing NSCs but not astrocytes. (E) f-BRAF expression increased tuberin phosphorylation in NSCs but not astrocytes. $\alpha$-Tubulin served as an internal loading control. (F) Increased tuberin membrane localization was observed in astrocytes compared with NSCs. Calnexin served as a marker for the membrane fraction. (C) Cytosol; (M) membrane; (Veh) vehicle. Error bars denote mean \pm SD. (NS) Not significant.

membrane-bound tuberin, preventing its phosphorylation and subsequent inactivation (Distefano et al. 2009; Dere et al. 2010). We found that cerebellar astrocytes express 20fold more polycystin-1 relative to their NSC counterparts (Supplemental Fig. S3I), potentially implicating polycystin-1 in the regulation of tuberin subcellular localization in astrocytes. However, shRNA-mediated polycystin-1 knockdown in astrocytes did not alter tuberin localization in wildtype astrocytes (Supplemental Fig. S3J), increase tuberin phosphorylation, or increase f-BRAF-expressing astrocyte proliferation (Supplemental Fig. S3K), excluding polycystin-1 as the molecular etiology responsible for the cell typespecific effects of f-BRAF in astrocytes and NSCs. It is likely that other mechanisms account for the differential subcellular localization of tuberin relevant to its inactivation by phosphorylation.

Tuberin phosphorylation leads to loss of tuberin/hamartin complex inhibition of Rheb and subsequent increased Rhebmediated mTOR activation (Castro et al. 2003; Inoki et al. 2003). We next sought to determine whether f-BRAF regulates $\mathrm{mTOR}$ activation in a tuberin/Rheb-dependent manner in NSCs. First, to confirm that TSC complex inactivation was responsible for f-BRAF-mediated mTOR activation, we expressed f-BRAF in wild-type or Tsc1 $1^{-1-}$ NSCs. Compared with their wild-type counterparts, empty vector-expressing Tsc1 ${ }^{-/-}$ NSCs exhibited a 2.5-fold increase in S6 phosphorylation as well as increased NSC proliferation (1.5-fold increase in cell number and $23 \%$ increase in neurosphere diameter). However, f-BRAF expression did not further increase S6 phosphorylation or proliferation in $\mathrm{Tsc1}^{-1-}$ NSCs (Fig. 4A,B). Second, ectopic Rheb expression in NSCs using a conditional Rheb-expressing mouse strain (Banerjee et al. 2011) resulted in a 2.1fold increase in S6 activation (Supplemental Fig. S4A) and increased NSC proliferation (1.4-fold in cell number; $23 \%$ increase in neurosphere diameter) (Supplemental Fig. S4B). Third, to determine whether f-BRAF regulation of mTOR required Rheb activation, Rheb expression was reduced by shRNA-mediated knockdown. Attenuated Rheb expression (70\% reduction) using two different shRNA constructs in f-BRAF-transduced NSCs reduced S6 phosphorylation and proliferation to wild-type levels with no effect on wild-type NSCs (Fig. 4C,D; Supplemental Fig. S4C). Together, these results establish TSC/Rheb-mediated mTOR activation as the mechanism underlying f-BRAF-mediated growth regulation of cerebellar NSCs.

Since mTOR can regulate cell growth in either an mTORC1-dependent (4E-BP1 and STAT3) or mTORC2-dependent (AKT) manner, we examined the activation state of each of these downstream signaling molecules. No changes in 4E-BP1 (Thr37/46), STAT3 (Ser727), or AKT (Ser473) were observed following f-BRAF expression in cerebellar NSCs (data not shown). mTOR can also regulate proliferation and cell cycle progression by increasing p27 phosphorylation and reducing total p27 expression (Hong et al. 2008; Dalvai et al. 2010). Following f-BRAF expression, there was increased p27 phosphorylation (Thr187) and decreased total p27 expression (Supplemental Fig. S4D). Moreover, both MEK (PD901) and mTOR (rapamycin) inhibition reduced p27 phosphorylation and restored $\mathrm{p} 27$ protein expression to wild-type levels (Supplemental Fig. S4D), indicating that f-BRAF controls NSC proliferation via mTOR-dependent regulation of p27 levels.

To elucidate the mechanism underlying f-BRAF regulation of p27 expression in cerebellar NSCs, we examined the mTOR downstream target S6K. Human S6K1 (hS6K1) expression increased NSC proliferation as well as p27 phosphorylation and decreased p27 protein levels (Supplemental Fig. S4E,F). In addition, S6K1 knockdown using two different shRNA constructs abrogated the f-BRAF-mediated increase in cell proliferation, S6 activation, and p27 phosphorylation and restored p27 expression to wild-type levels (Fig. 4E,F; Supplemental Fig. S4G). To assess whether p27 phosphorylation required cyclin-dependent kinase 
A

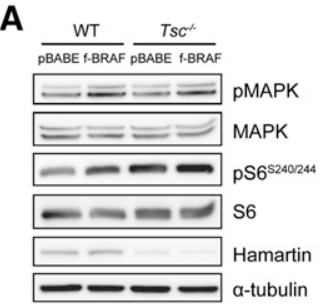

C

E
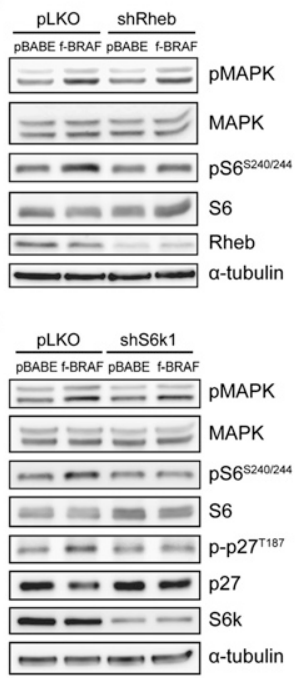

G

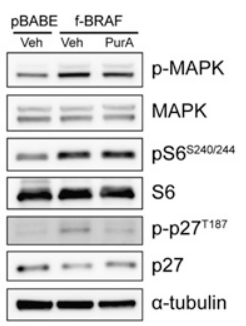

B

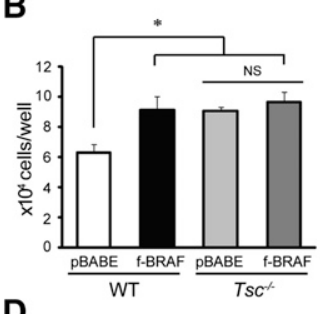

D

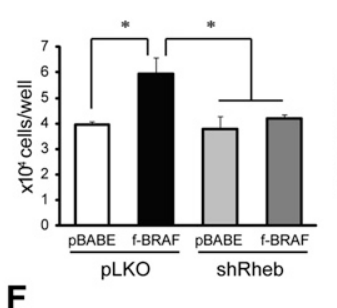

$\mathbf{F}$
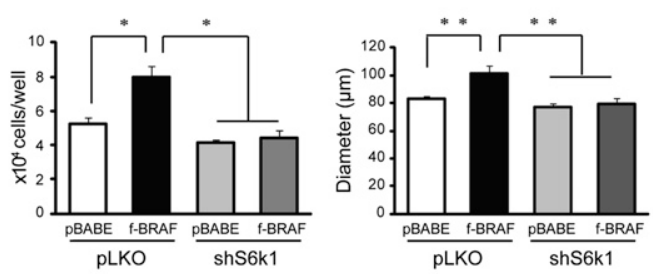

H
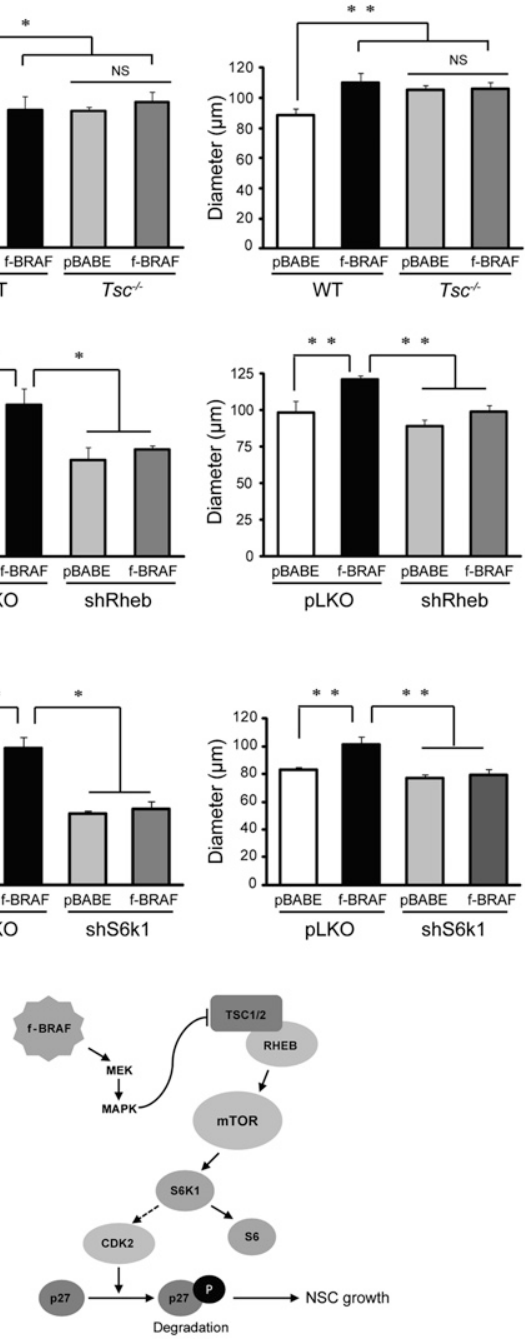

Materials and methods

Mice

Tsc1 $1^{\text {flox/llox }}$ mice (Uhlmann et al. 2002) and conditional Rhebexpressing transgenic mice (Banerjee et al. 2011) were generated as previously described. All mice were maintained on a C57BL/6 background and used in accordance with an approved Animal Studies protocol at Washington University.

\section{Human tumor samples}

Tumor specimens were obtained from the Tissue Procurement Core Facility at Washington University under an approved Institutional Review Board protocol.

\section{Primary astrocyte and NSC cultures}

Cerebellar hemispheres were microdissected from the brains of C57BL/6 mouse pups to establish primary astrocyte and NSC cultures, and proliferation assays were performed as previously described (Dasgupta and Gutmann 2005; Lee et al. 2010; Banerjee et al. 2011). Wild-type and Tsc1 $1^{-1-}$ NSCs were generated from $T s c 1^{\text {flox/flox }}$ pups following infection with LacZ and Cre adenovirus, respectively. Retroviral and lentiviral (Supplemental Table 1) transduction was performed (Lee et al. 2010) for overexpression and knockdown studies, respectively. Expression of the $f-B R A F$ transgene was verified by RT-PCR (Lee et al. 2012). All in vitro experiments were performed at least three times using primary NSC and astrocyte cultures generated using pups from different litters and were independently transduced with the respective constructs.

\section{Subcellular fractionation}

Following hypotonic (10 mM HEPES at $\mathrm{pH} 7.9,1.5 \mathrm{mM}$ $\mathrm{MgCl}_{2}, 10 \mathrm{mM} \mathrm{KCl}$, protease inhibitors) lysis and disruption of cells with a 30-gauge syringe needle, crude nuclei were

2 (Cdk2) (Montagnoli et al. 1999), f-BRAF-expressing NSCs were treated with the Cdk2 inhibitor purvalanol-A (PurA). Following PurA treatment, f-BRAF-induced cerebellar NSC proliferation (Supplemental Fig. S4H) and p27 phosphorylation were restored to wild-type levels with no effect on mTOR activation (Fig. 4G). Collectively, these results establish that f-BRAF regulation of NSC proliferation requires mTOR-mediated activation of S6K1 to suppress $\mathrm{p} 27$ levels in a Cdk2-dependent manner (Fig. 4H).

The finding that f-BRAF regulates NSC growth in an mTOR-dependent manner establishes a central growth control target for both sporadic (KIAA1549:BRAF) and familial (NF1-associated) pediatric low-grade glioma and provides compelling preclinical evidence for the use of pelleted by centrifugation, and supernatants were subjected to ultracentrifugation at $100,000 \mathrm{~g}$ for $1 \mathrm{~h}$ at $4^{\circ} \mathrm{C}$ to generate cytosolic and membrane fractions. Equal percentages of each fraction were subjected to SDS-PAGE and immunoblot analysis.

\section{Western blotting}

Cell lysis, SDS-PAGE, and immunoblot analysis were performed as previously reported (Uhlmann et al. 2004).

\section{Intracranial injections}

Three-week-old wild-type C57/BL6 mice were anesthetized by an intraperitoneal (i.p.) injection of $100 \mathrm{mg} / \mathrm{kg}$ ketamine and $6 \mathrm{mg} / \mathrm{kg}$ xylazine. NSCs $\left(5 \times 10^{5}\right)$ in $2 \mu \mathrm{L}$ of PBS were injected into the cerebellum using 
Kaul et al.

a Hamilton syringe $3 \mathrm{~mm}$ below the dura mater using a stereotaxic apparatus. Mice were euthanized 2.5 and 6 mo later.

\section{Immunostaining}

Paraffin or frozen sections were processed (Dasgupta and Gutmann 2005) prior to staining with appropriate antibodies (Supplemental Table 2).

\section{Statistical analysis}

All in vitro experiments were analyzed using the Student's $t$-test. Statistical significance was set at $P<0.05$.

\section{Acknowledgments}

We thank Crystal White-Worsena for technical assistance. This work was funded by grants from the National Institutes of Health (NS065547) and National Brain Tumor Society to D.H.G. Y.H.C. is supported by a fellowship from the American Brain Tumor Association.

\section{References}

Alcantara Llaguno S, Chen J, Kwon CH, Jackson EL, Li Y, Burns DK Alvarez-Buylla A, Parada LF. 2009. Malignant astrocytomas originate from neural stem/progenitor cells in a somatic tumor suppressor mouse model. Cancer Cell 15: 45-56.

Babchia N, Calipel A, Mouriaux F, Faussat AM, Mascarelli F. 2010. The $\mathrm{PI} 3 \mathrm{~K} /$ Akt and mTOR/P70S6K signaling pathways in human uveal melanoma cells: Interaction with B-Raf/ERK. Invest Ophthalmol Vis Sci 51: 421-429.

Banerjee S, Crouse NR, Emnett RJ, Gianino SM, Gutmann DH. 2011. Neurofibromatosis-1 regulates mTOR-mediated astrocyte growth and glioma formation in a TSC/Rheb-independent manner. Proc Natl Acad Sci 108: 15996-16001.

Carriere A, Cargnello M, Julien LA, Gao H, Bonneil E, Thibault P, Roux PP 2008. Oncogenic MAPK signaling stimulates mTORC1 activity by promoting RSK-mediated raptor phosphorylation. Curr Biol 18: 1269-1277.

Castro AF, Rebhun JF, Clark GJ, Quilliam LA. 2003. Rheb binds tuberous sclerosis complex 2 (TSC2) and promotes S6 kinase activation in a rapamycin- and farnesylation-dependent manner. J Biol Chem 278: 32493-32496.

Central Brain Tumor Registry of the United States. 2012. 2012 CBTRUS statistical report: Primary brain and central nervous system tumors diagnosed in the United States in 2004-2008. http://www.cbtrus.org/ 2012-NPCR-SEER/CBTRUS_Report_2004-2008_3-23-2012.pdf.

Dalvai M, Schubart K, Besson A, Matthias P. 2010. Oct1 is required for mTOR-induced G1 cell cycle arrest via the control of p27(Kip1) expression. Cell Cycle 9: 3933-3944.

Dasgupta B, Gutmann DH. 2005. Neurofibromin regulates neural stem cell proliferation, survival, and astroglial differentiation in vitro and in vivo. J Neurosci 25: 5584-5594.

Dasgupta B, Yi Y, Chen DY, Weber JD, Gutmann DH. 2005. Proteomic analysis reveals hyperactivation of the mammalian target of rapamycin pathway in neurofibromatosis 1-associated human and mouse brain tumors. Cancer Res 65: 2755-2760.

Dere R, Wilson PD, Sandford RN, Walker CL. 2010. Carboxy terminal tail of polycystin-1 regulates localization of TSC2 to repress mTOR. PLOS ONE 5: e9239. doi: 10.1371/journal.pone.0009239.

Distefano G, Boca M, Rowe I, Wodarczyk C, Ma L, Piontek KB, Germino GG, Pandolfi PP, Boletta A. 2009. Polycystin-1 regulates extracellular signal-regulated kinase-dependent phosphorylation of tuberin to control cell size through mTOR and its downstream effectors S6K and 4EBP1. Mol Cell Biol 29: 2359-2371.

Duong MT, Akli S, Wei C, Wingate HF, Liu W, Lu Y, Yi M, Mills GB, Hunt KK, Keyomarsi K. 2012. LMW-E/CDK2 deregulates acinar morphogenesis, induces tumorigenesis, and associates with the activated b-Raf-ERK1/2-mTOR pathway in breast cancer patients. PLoS Genet 8: e1002538. doi: 10.1371/journal.pgen.1002538.

Faustino A, Couto JP, Populo H, Rocha AS, Pardal F, Cameselle-Teijeiro JM, Lopes JM, Sobrinho-Simoes M, Soares P. 2012. mTOR pathway overactivation in BRAF mutated papillary thyroid carcinoma. J Clin Endocrinol Metab 9: E1139-E1149. doi: 10.1210/jc.2011-2748.
Gronych J, Korshunov A, Bageritz J, Milde T, Jugold M, Hambardzumyan D, Remke M, Hartmann C, Witt H, Jones DT, et al. 2011. An activated mutant BRAF kinase domain is sufficient to induce pilocytic astrocytoma in mice. J Clin Invest 121: 1344-1348.

Hong F, Larrea MD, Doughty C, Kwiatkowski DJ, Squillace R, Slingerland JM. 2008. mTOR-raptor binds and activates SGK1 to regulate p27 phosphorylation. Mol Cell 30: 701-711.

Inoki K, Li Y, Xu T, Guan KL. 2003. Rheb GTPase is a direct target of TSC2 GAP activity and regulates mTOR signaling. Genes Dev 17: 1829-1834.

Jacob K, Albrecht S, Sollier C, Faury D, Sader E, Montpetit A, Serre D, Hauser P, Garami M, Bognar L, et al. 2009. Duplication of $7 q 34$ is specific to juvenile pilocytic astrocytomas and a hallmark of cerebellar and optic pathway tumours. Br J Cancer 101: 722-733.

Jacob K, Quang-Khuong DA, Jones DT, Witt H, Lambert S, Albrecht S, Witt O, Vezina C, Shirinian M, Faury D, et al. 2011. Genetic aberrations leading to MAPK pathway activation mediate oncogeneinduced senescence in sporadic pilocytic astrocytomas. Clin Cancer Res 17: 4650-4660.

Jones DT, Kocialkowski S, Liu L, Pearson DM, Backlund LM, Ichimura K, Collins VP. 2008. Tandem duplication producing a novel oncogenic BRAF fusion gene defines the majority of pilocytic astrocytomas. Cancer Res 68: 8673-8677.

Kluwe L, Hagel C, Tatagiba M, Thomas S, Stavrou D, Ostertag H, von Deimling A, Mautner VF. 2001. Loss of NF1 alleles distinguish sporadic from NF1-associated pilocytic astrocytomas. I Neuropathol Exp Neurol 60: 917-920.

Lee DY, Yeh TH, Emnett RJ, White CR, Gutmann DH. 2010. Neurofibromatosis-1 regulates neuroglial progenitor proliferation and glial differentiation in a brain region-specific manner. Genes Dev 24: 2317-2329.

Lee DY, Gianino SM, Gutmann DH. 2012. Innate neural stem cell heterogeneity determines the patterning of glioma formation in children. Cancer Cell 22: 131-138.

Liu C, Sage JC, Miller MR, Verhaak RG, Hippenmeyer S, Vogel H, Foreman O, Bronson RT, Nishiyama A, Luo L, et al. 2011. Mosaic analysis with double markers reveals tumor cell of origin in glioma. Cell 146: 209-221.

Lyustikman Y, Momota H, Pao W, Holland EC. 2008. Constitutive activation of Raf-1 induces glioma formation in mice. Neoplasia 10: 501-510.

Ma L, Chen Z, Erdjument-Bromage H, Tempst P, Pandolfi PP. 2005. Phosphorylation and functional inactivation of TSC2 by Erk implications for tuberous sclerosis and cancer pathogenesis. Cell 121: 179-193.

Montagnoli A, Fiore F, Eytan E, Carrano AC, Draetta GF, Hershko A, Pagano M. 1999. Ubiquitination of $\mathrm{p} 27$ is regulated by Cdk-dependent phosphorylation and trimeric complex formation. Genes Dev 13: 1181-1189.

Ohgaki H, Kleihues P. 2005. Population-based studies on incidence, survival rates, and genetic alterations in astrocytic and oligodendroglial gliomas. J Neuropathol Exp Neurol 64: 479-489.

Pfister S, Janzarik WG, Remke M, Ernst A, Werft W, Becker N, Toedt G, Wittmann A, Kratz C, Olbrich H, et al. 2008. BRAF gene duplication constitutes a mechanism of MAPK pathway activation in low-grade astrocytomas. J Clin Invest 118: 1739-1749.

Raabe EH, Lim KS, Kim JM, Meeker A, Mao XG, Nikkhah G, Maciaczyk J, Kahlert U, Jain D, Bar E, et al. 2011. BRAF activation induces transformation and then senescence in human neural stem cells: A pilocytic astrocytoma model. Clin Cancer Res 17: 3590-3599.

Robinson JP, VanBrocklin MW, Guilbeault AR, Signorelli DL, Brandner S, Holmen SL. 2010. Activated BRAF induces gliomas in mice when combined with Ink4a/Arf loss or Akt activation. Oncogene 29: 335-344.

Taylor MD, Poppleton H, Fuller C, Su X, Liu Y, Jensen P, Magdaleno S, Dalton J, Calabrese C, Board J, et al. 2005. Radial glia cells are candidate stem cells of ependymoma. Cancer Cell 8: 323-335.

Uhlmann EJ, Wong M, Baldwin RL, Bajenaru ML, Onda H, Kwiatkowski DJ, Yamada K, Gutmann DH. 2002. Astrocyte-specific TSC1 conditional knockout mice exhibit abnormal neuronal organization and seizures. Ann Neurol 52: 285-296.

Uhlmann EJ, Li W, Scheidenhelm DK, Gau CL, Tamanoi F, Gutmann DH. 2004. Loss of tuberous sclerosis complex 1 (Tsc1) expression results in increased Rheb/S6K pathway signaling important for astrocyte cell size regulation. Glia 47: 180-188. 


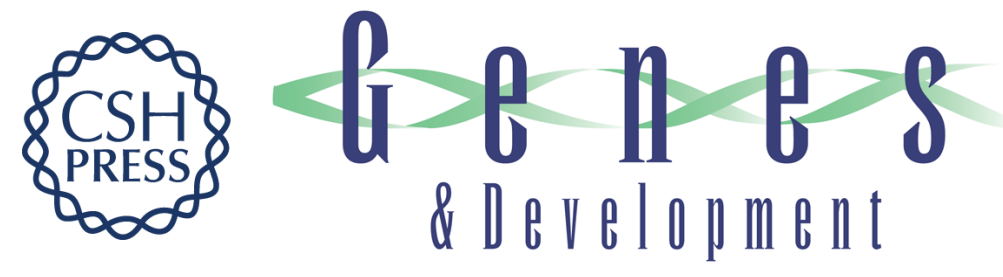

\section{Pediatric glioma-associated KIAA1549:BRAF expression regulates neuroglial cell growth in a cell type-specific and mTOR-dependent manner}

Aparna Kaul, Yi-Hsien Chen, Ryan J. Emnett, et al.

Genes Dev. 2012, 26: originally published online November 14, 2012

Access the most recent version at doi:10.1101/gad.200907.112

Supplemental Material

References

License

Email Alerting

Service
http://genesdev.cshlp.org/content/suppl/2012/11/12/gad.200907.112.DC1

This article cites 32 articles, 12 of which can be accessed free at: http://genesdev.cshlp.org/content/26/23/2561.full.html\#ref-list-1

Receive free email alerts when new articles cite this article - sign up in the box at the top right corner of the article or click here.

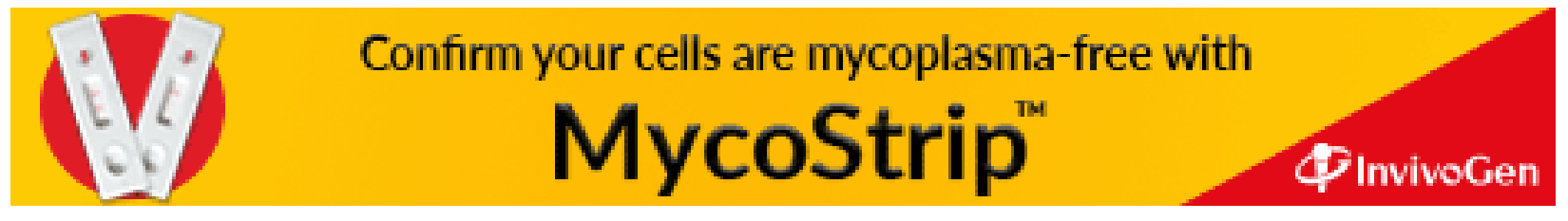

